\title{
An Enzymatic Basis for Lewis Blood Types in Man
}

\author{
Evelyn F. Grollman, Akira Kobata, and Victor Ginsburg \\ From the National Institute of Arthritis and Metabolic Diseases, National \\ Institutes of Health, Bethesda, Maryland 20014
}

A B S T A C T Milk from women with blood type $\operatorname{Le}(\mathrm{a}+)$ or $\operatorname{Le}(\mathrm{b}+)$ contains a specific fucosyltransferase not found in the milk of women with blood type Le $(a-b-)$. The enzyme, a guanosine diphosphate L-fucose: $N$-acetyl- $\beta$-D-glucosaminylsaccharide $\alpha$-4-L-fucosyltransferase is apparently required for the synthesis of the structural determinants of $\mathrm{Le}^{\mathrm{a}}$ and $\mathrm{Le}^{\mathrm{b}}$ specificity, both of which contain fucose in an $\alpha-1,4$ linkage to $N$-acetylglucosamine. The same enzyme is also involved in the synthesis of milk oligosaccharides, as two oligosaccharides which contain this linkage are absent from the milk of women with $\operatorname{Le}(\mathrm{a}-\mathrm{b}-)$ blood type.

\section{INTRODUCTION}

The Lewis blood types discovered by Mourant (2) and by Andresen (3) are defined by two antigens, Le ${ }^{a}$ and $\mathrm{Le}^{\mathrm{b}}$, and their antibodies, anti-Le ${ }^{\mathbf{a}}$ and anti-Le ${ }^{\mathbf{b}}$. Approximately $20 \%$ of Caucasians have the blood type Le $(\mathrm{a}+)$; $73 \%$ have the blood type $\mathrm{Le}(\mathrm{b}+)$; the remaining $7 \%$ have the blood type $\operatorname{Le}(a-b-)(4)$.

The antigenic determinant for the $\mathrm{Le}^{\mathrm{a}}$ specificity of soluble blood group substances is a carbohydrate with the structure $(5-7)$ :

$$
\begin{gathered}
\alpha \text {-L-Fuc- }(1 \rightarrow 4) \\
\beta \text {-D-Gal- }(1 \rightarrow 3)-3-\mathrm{D}-G \mathrm{ilc} \text { - Ac-R }
\end{gathered}
$$

The $\mathrm{Le}^{\mathrm{b}}$ determinant has the same structure with an additional L-fucose in an $\alpha-1,2$ linkage to D-galactose $(7,8)$ :

$$
\alpha \text {-L-Fuc- }(1 \rightarrow 2)-\beta \text {-D-Gal-Fuc- }(1 \rightarrow 4)
$$

The addition of this second L-fucose is catalyzed by a specific L-fucosyltransferase present in milk from individuals who secrete soluble blood group substances $A$, $\mathrm{B}$, or $\mathrm{O}(\mathrm{H})$ corresponding to their particular $\mathrm{ABO}$

Part of this work was presented at the 1968 meeting of the American Society of Biological Chemists (1).

Received for publication 20 Fcbruary 1969 and in revised form 14 April 1969. blood type ("secretors") and is absent from milk of those who do not ("nonsecretors") (9). The absence of this enzyme from "nonsecretors" explains the fact that they never have the blood type $\operatorname{Le}(b+)(10)$, as they would be unable to synthesize the Leb determinant shown above.

Both Le $\mathrm{Le}^{\mathrm{a}}$ and $\mathrm{Le}^{\mathrm{b}}$ determinants contain $\mathrm{L}$-fucose in an $\alpha-1,4$ linkage to $N$-acetyl-D-glucosamine. As hypothesized by Watkins and Morgan, the Le gene is responsible for the formation of an enzyme that catalyzes the addition of this L-fucose (11-12). Individuals with the genotype lele would lack the enzyme and have the blood type Le $(a-b-)$, as they would be unable to synthesize either structure. The present paper describes an enzyme in human milk which catalyzes the addition of $\mathrm{L}$-fucose from guanosine diphosphate L-fucose to $N$-acetyl-D-glucosamine residues. The enzyme appears to be the hypothetical product of the $L e$ gene, as it is found only in milk from donors with blood type $\mathrm{Le}(\mathrm{a}+)$ or Le $(b+)$ and is absent in milk from donors with blood type $\operatorname{Le}(\mathrm{a}-\mathrm{b}-)$. This distribution provides an enzymatic basis for blood type $\operatorname{Le}(a-b-)$.

\section{METHODS}

Materials. GDP-L-fucose $-{ }^{14} \mathrm{C}\left(2.6 \times 10^{7} \mathrm{cpm} / \mu \mathrm{mole}\right)$ and GDP-L-fucose were synthesized enzymatically from GDPD-mannose $-{ }^{14} \mathrm{C}$ and GDP-D-mannose (13). Lacto- $N$-fucopentaose I (2-O- $\alpha$-Fuc-3-O- $\beta$-Gal-3-O- $\beta$-GlcNAc-4-O- $\beta-\mathrm{Gal}$ Glc), lacto- $N$-fucopentaose II $(3-O-\beta-\mathrm{Gal}-[4-O-\alpha-\mathrm{Fuc}]-3-$ $O-\beta$-GlcNAc-4- $O-\beta$-Gal-Glc), and lacto- $N$-difucohexaose I $\quad(2-O-\alpha-\mathrm{Fuc}-3-O-\beta-\mathrm{Gal}-[4-O-\alpha-\mathrm{Fuc}]-3-O-\beta-\mathrm{GlcN} A \mathrm{c}-4-O-$ $\beta$-Gal-Glc) were generously provided by Dr. Adeline Gaulıe of the Max Planck Institute, Heidelberg.

Enzyme preparation. Milk was obtained from donors and frozen until used. The Lewis blood type of the donors was determined on saliva and red cells by standard techniques (14). For the enzyme studies, milk samples $(10 \mathrm{ml})$ were centrifuged at $105,000 \mathrm{~g}$ for $1 \mathrm{hr}$ and the congealed fat removed with a spatula. The supernatant fluid was put on a $2 \times 25 \mathrm{~cm}$ column of Sephadex G25 previously equilibrated with $0.05 \mathrm{M}$ Tris buffer, $\mathrm{pH} 7$, and $0.005 \mathrm{~m}$ glutathione, and the column was eluted with the same buffer. The fractions containing protein (as determined by absorption at $280 \mathrm{~m} \mu$ ) were pooled, and ammonium sulfate was addied to $80 \%$ saturation. The precipitate was collected by centrifugation, 
TABLE 1

Absence of Oligosaccharides that Contain Fucose-Linked $\alpha-1,4$ to $N$-Acetylglucosamine from Milk of Donors with Le $(a-b-)$ Blood Type

\begin{tabular}{|c|c|c|c|c|c|}
\hline \multirow[b]{3}{*}{ Donor } & \multirow{2}{*}{\multicolumn{2}{|c|}{ Blood type }} & \multicolumn{3}{|c|}{ Oligosaccharide } \\
\hline & & & \multirow{2}{*}{$\begin{array}{l}\text { Lacto- } N \text {-fuco- } \\
\text { pentaose I }\end{array}$} & \multirow{2}{*}{$\begin{array}{l}\text { Lacto- } N \text {-fuco- } \\
\text { pentaose II }\end{array}$} & \multirow{2}{*}{$\begin{array}{l}\text { Lacto- } N \text {-difuco- } \\
\text { hexaose I }\end{array}$} \\
\hline & $\mathrm{ABO}$ & Lewis & & & \\
\hline & & & \multicolumn{3}{|c|}{ $\mu$ moles $/ \mathrm{ml}$} \\
\hline E. K. & $\mathrm{A}_{2}$ & $\operatorname{Le}(a-b-)$ & 0.81 & $<0.01$ & $<0.02$ \\
\hline C. R. & 0 & $\operatorname{Le}(a-b-)$ & 2.68 & $<0.01$ & $<0.02$ \\
\hline O. R. & 0 & $\operatorname{Le}(a-b-)$ & 2.29 & $<0.01$ & $<0.02$ \\
\hline J. S. & 0 & $\operatorname{Le}(a+)$ & $<0.01^{*}$ & 0.41 & $<0.02^{*}$ \\
\hline D. R. & 0 & $\operatorname{Le}(\mathrm{a}+)$ & $<0.01^{*}$ & 0.51 & $<0.02^{*}$ \\
\hline J. C. & $A_{1} B$ & $\operatorname{Le}(b+)$ & 0.05 & 0.11 & $0.23 \ddagger$ \\
\hline G. H. & B & $\operatorname{Le}(b+)$ & 0.03 & 0.04 & $0.13 \ddagger$ \\
\hline L. N. & 0 & $\operatorname{Le}(b+)$ & 0.13 & 0.20 & $0.52 \ddagger$ \\
\hline
\end{tabular}

8- $\mathrm{ml}$ of milk from individual donors, from which lactose had been partially removed by precipitation with $60 \%$ alcohol in the cold, was separated on a Sephadex G25 column as described previously (17). The fraction which contained the penta- and hexasaccharides was reduced in volume in vacuo and applied as a 6 inch band on Whatman No. 1 paper and chromatographed for $140 \mathrm{hr}$ in solvent $\mathrm{A}$. The areas of paper corresponding to standard lacto- $N$-fucopentaose I, lacto- $N$-fucopentaose II, and lacto- $N$-difucohexaose I were cut and eluted with $1 \mathrm{ml}$ of water, and the oligosaccharide content of the eluate was determined as described in Methods.

* The donors with Le $(\mathrm{a}+)$ blood type are all "nonsecretors" (24) and their milk does not contain oligosaccharides with Fuc- $\alpha-(1 \rightarrow 2)-$ Gal groupings $(17,19)$.

$\ddagger$ The values given for lacto- $N$-difucohexaose I are maximal, as they also include small amounts of lacto- $N$-difucohexaose II which is only partially separated from lacto- $N$-difucohexaose I by paper chromatography. No lacto- $N$-difucohexaose I could be detected chromatographically in milk from donors with Le $(a+)$ or Le $(a-b-)$ blood type. The limit of detection by this method is approximately $0.02 \mu$ moles of oligosaccharide per $\mathrm{ml}$ of milk.

dissolved in $5 \mathrm{ml}$ of Tris-glutathione buffer, the resulting solution dialyzed overnight against 1 liter of the same buffer and used for the experiments described in this paper.

Chromatography. Descending paper chromatography was carried out using the following solvents: (A) ethyl acetatepyridine-water $(2: 1: 2)$, upper layer; (B) 1-propanol-ethyl acetate-water $(7: 1: 2)$; (C) 1-butanol-pyridine-water (6:4:3) ; and (D) ethyl acetate-pyridine-acetic acid-water $(5: 5: 1: 3)$. Sugars were located on paper with $\mathrm{AgNO}_{3}$ reagent (15).

Quantitative analysis of oligosaccharides. Oligosaccharides were isolated by paper chromatography and determined colorimetrically. Lacto- $N$-fucopentaose I and lacto- $N$-difucohexaose I were estimated by analysis for fucose using cysteine-sulfuric acid reagent (16). Lacto- $N$-fucopentaose II could not be determined by its fucose content, because samples isolated from different sources of milk contain variable amounts of an isomeric fucose-containing pentasaccharide (17). Unlike lacto- $N$-fucopentaose II, however, this isomer does not react with the Elson-Morgan reagent (18) for hexosamine after liberation of fucose by mild acid hydrolysis. Accordingly, lacto- $N$-fucopentaose II was determined by the Elson-Morgan assay for hexosamine (18) after hydrolysis of the oligosaccharide fraction with 0.01 $N \mathrm{HCl}$ for $40 \mathrm{~min}$ at $100^{\circ} \mathrm{C}$. Authentic oligosaccharides were used as standards.

\section{RESULTS}

Oligosaccharides in milk from $\operatorname{Le}(a-b-)$ donors. Milk of "nonsecretors" lacks all the oligosaccharides that contain L-fucose in an $\alpha-1,2$ linkage to D-galactose including 2 -fucosyllactose, lactodifucotetraose, lacto- $N$ fucopentaose I, and lacto- $N$-difucohexaose I $(17,19)$, indicating that enzymes involved in the synthesis of blood group substances also play a role in the formation of milk oligosaccharides. Two of the known oligosaccharides of human milk, lacto- $N$-fucopentaose II and lacto- $N$-difucohexaose I (20-23), contain L-fucose in an $\alpha-1,4$ linkage to $N$-acetyl-D-glucosamine. If $\operatorname{Le}(\mathrm{a}-$ $\mathrm{b}-$ ) blood type did indeed result from an inability to form this linkage, then milk from donors of blood type Le $(a-b-)$ might be expected to lack both oligosaccharides. This was found to be the case as shown in Table I. The three samples of milk from $\operatorname{Le}(a-b-)$ individuals did not contain detectable amounts of either oligosaccharide in contrast to the results obtained with milk from $\operatorname{Le}(\mathrm{a}+)$ or $\operatorname{Le}(\mathrm{b}+)$ individuals which are included in Table I for comparison. The level of lacto- $N$ fucopentaose I, which is the probable precursor of lacto$\mathrm{N}$-difucohexaose I, was considerably higher in milk from the three $\operatorname{Le}(\mathrm{a}-\mathrm{b}-)$ individuals than in milk from seven $\operatorname{Le}(\mathrm{b}+)$ individuals $(17)$.

Transfer of L-fucose to lacto- $N$-fucopentaose $I$. L-fucosyltransferases present in human milk transfer L-fucose from GDP-L-fucose to several oligosaccharides including lacto- $N$-fucopentaose I (9). The product formed when lacto- $N$-fucopentaose $I$ is used as an acceptor has 


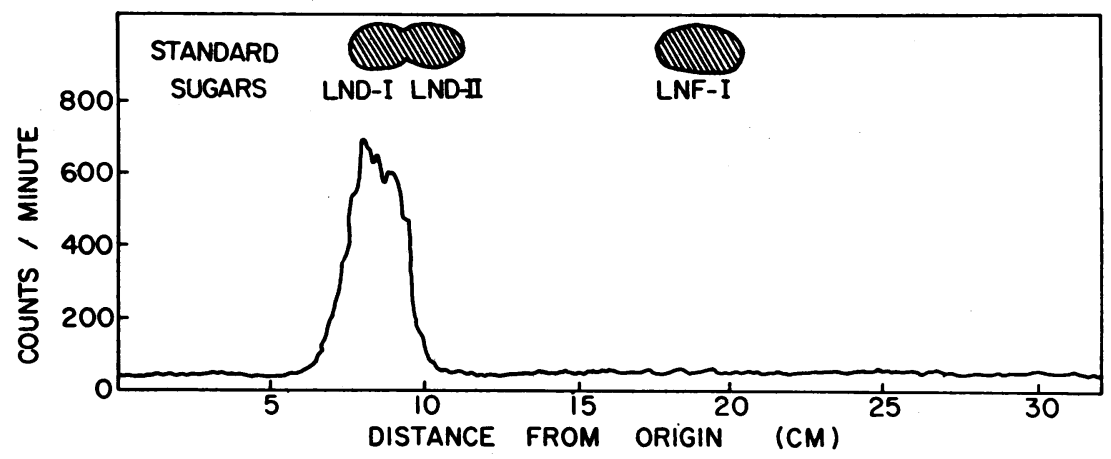

Figure 1 Addition of $\mathrm{L}$-fucose- ${ }^{14} \mathrm{C}$ to lacto- $N$-fucopentaose $\mathrm{I}$. The labeled oligosaccharide was prepared as follows : to $0.25 \mu$ mole of Tris buffer, $\mathrm{pH} 7.0$, in a final volume of $50 \mu 1$, was added $1.5 \mathrm{~m} \mu$ mole of guanosine diphosphate-L-fucose- ${ }^{14} \mathrm{C}$ $\left(2.7 \times 10^{4} \mathrm{cpm} / \mathrm{m} \mu \mathrm{mole}\right), 0.1 \mu$ mole of lacto- $N$-fucopentaose $\mathrm{I}, 0.25 \mu$ mole of $\mathrm{MnCl}_{2}$, and $20 \mu 1$ of Sephadex-treated milk from donor S. L. who had the blood type $\mathrm{Le}\left(\mathrm{b}^{+}\right)$. After incubation at $37^{\circ} \mathrm{C}$ for $5 \mathrm{hr}$, the reaction was stopped by heating at $100^{\circ} \mathrm{C}$ for $1 \mathrm{~min}$. The reaction mixture was deionized by passage through an ion-exchange column containing $1 \mathrm{ml}$ of equal parts of Bio-Rad AG $3 \times 4 \mathrm{COH}^{-}$ form) and Bio-Rad AG 50W $\times 8$ ( $\mathrm{H}^{+}$form). The neutral oligosaccharides in the effluent were chromatographed as a $1 \frac{1}{2}$ inch band in solvent $A$ for $140 \mathrm{hr}$ and the resulting chromatogram scanned for ${ }^{11} \mathrm{C}$ activity with the result shown above. No product was formed if lacto- $N$-fucopentaose was omitted from the incubation mixture. The standard sugars are lacto- $N$-difucohexaose I (LND-I), lacto- $N$-difucohexaose II (LND-II), and lacto- $N$-fucopentaose I (LNF-I).

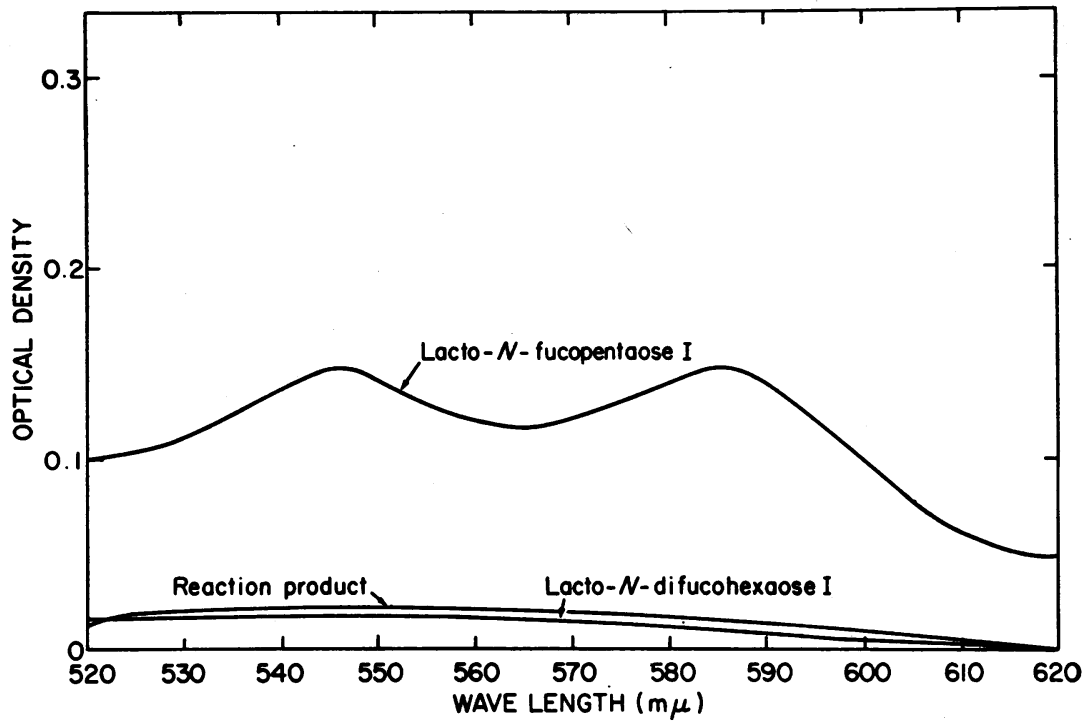

FIGURE 2 Absorption spectra exhibited by lacto- $N$-fucopentaose I, lacto- $N$-difucohexaose I, and the reaction product (5 $\mu \mathrm{g}$ of each) in the Elson-Morgan test for $N$-acetylaminosugars (18). The assays were run in a final volume of $0.2 \mathrm{ml}$ and the absorption spectra recorded with a Cary spectrophotometer.

The enzymatic product was prepared as follows : to $12.5 \mu$ moles of Tris buffer, $\mathrm{pH}$ 7.0 , in a final volume of $2.5 \mathrm{ml}$, was added $0.89 \mu$ mole of GDP-L-fucose- ${ }^{14} \mathrm{C}(40,000$ cpm), $2.5 \mu$ moles of lacto- $N$-fucopentaose I, $12.5 \mu$ moles of $\mathrm{MnCl}_{2}, 40 \mu$ of toluene, and $1 \mathrm{ml}$ of Sephadex-treated milk from donor L. N. who has the blood type Le $\left(b^{+}\right)$. After incubation for $26 \mathrm{hr}$ at $37^{\circ} \mathrm{C}$, the reaction mixture was treated as described in Fig. 1, except that the effluent was chromatographed as a 3 inch band. The area chromatographing with lacto- $N$-difucohexaose I was eluted with water and the amount of the product calculated from its radioactivity. $0.01 \mu$ mole of hexasaccharide $(10.2 \mu \mathrm{g})$ was obtained. 
the chromatographic mobility of lacto- $N$-difucohexaose I as shown in Fig. 1. The enzyme that catalyzes this reaction as shown in Table II is found in milk from $\mathrm{Le}(\mathrm{a}+)$ or $\mathrm{Le}(\mathrm{b}+)$ individuals and not in milk from $\operatorname{Le}(a-b-)$ individuals. The addition of milk from a $\operatorname{Le}(a-b-)$ individual to milk from $a \operatorname{Le}(a+)$ or $\mathrm{Le}(\mathrm{b}+)$ individual did not decrease the amount of product formed. All samples contain comparable amounts of the fucosyltransferase(s) that $\operatorname{add}(\mathrm{s})$ L-fucose to lactose.

Characterization of the product. $\mathrm{L}$-fucose occurs in several different linkages in the oligosaccharides of human milk: $\alpha-1,2$ to D-galactose (22); $\alpha-1,3$ to D-glucose $(25,26)$; and $\alpha-1,3(17)$ and $\alpha-1,4(20,25)$ to $N$-acetylD-glucosamine. It is probable that the fucosyltransferase with which we are concerned in the present paper adds L-fucose to lacto- $N$-fucopentaose I only in an $\alpha-1,4$ linkage to the $N$-acetyl-D-glucosamine residue to form lacto$N$-difucohexaose I. Besides the correlation of the occurrence of the enzyme with Lewis blood type (Table II) as well as with the occurrence of lacto- $N$-fucopentaose II and lacto- $N$-difucohexaose I which contain this linkage (Table I), the following additional evidence favors the above mentioned hypothesis:

(a) The product behaved chromatographically like

TABLE II

Fucosyltransferase Activity of Human Milk

\begin{tabular}{|c|c|c|c|}
\hline \multirow[b]{2}{*}{ Donor } & \multirow[b]{2}{*}{$\begin{array}{l}\text { Lewis blood type } \\
\text { of donor }\end{array}$} & \multicolumn{2}{|c|}{ Fucose transferred } \\
\hline & & $\begin{array}{c}\text { To lacto- } \\
N \text {-fuco- } \\
\text { pentaose } \\
\text { I }\end{array}$ & To lactose \\
\hline & & \multicolumn{2}{|c|}{$c p m$} \\
\hline J.S. & $\operatorname{Le}(a+)$ & 2310 & 810 \\
\hline D. $\mathrm{R}$. & $\operatorname{Le}(a+)$ & 1890 & 420 \\
\hline S. L. & $\mathrm{Le}(\mathrm{b}+)$ & 2480 & 610 \\
\hline L. N. & $\operatorname{Le}(b+)$ & 3060 & 930 \\
\hline J. C. & $\operatorname{Le}(b+)$ & 890 & 320 \\
\hline G. H. & $\operatorname{Le}(b+)$ & 1800 & 580 \\
\hline E. $\mathrm{K}$. & $\operatorname{Le}(a-b-)$ & 0 & 430 \\
\hline C.R. & Le $(a-b-)$ & 0 & 510 \\
\hline
\end{tabular}

Sephadex-treated milk samples from various donors were assayed for fucosyltransferase activity as described in Fig. 1 with either $0.1 \mu$ mole of lacto- $N$-fucopentaose I or $0.1 \mu$ mole of lactose as acceptors. After incubation for $5 \mathrm{hr}$ at $37^{\circ} \mathrm{C}$, the incubation mixtures were deionized and the neutral sugars chromatographed as a $1 \frac{1}{2}$ inch band on Whatman No. 3 paper with solvent A for $40 \mathrm{hr}$ when lactose was used, and $140 \mathrm{hr}$ when lacto- $N$-fucopentaose I was used. The ${ }^{14} \mathrm{C}$ activity was determined by counting appropriate sections of the chromatograms in a scintillation counter as previously described (9). A zero in the column means that the radioactivity in the area of the product did not differ significantly from background levels, which were approximately $30 \mathrm{cpm}$.
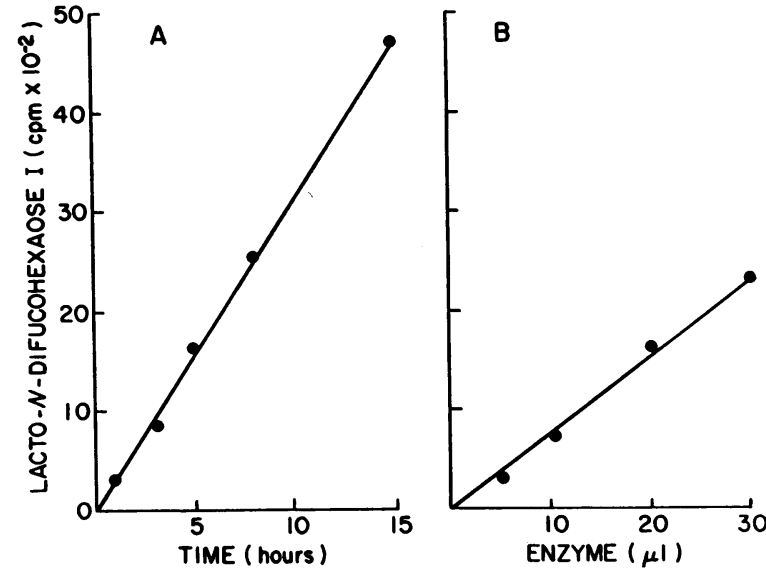

Figure 3 Effect of time and enzyme concentration on the formation of lacto- $N$-difucohexaose I. Reaction mixtures and conditions were the same as in Fig. 1, except that the time of incubation (A) or the amount of enzyme (B) was varied as indicated.

lacto- $N$-difucohexaose I in solvents $\mathrm{A}, \mathrm{B}, \mathrm{C}$, and $\mathrm{D}$ which distinguish lacto- $N$-difucohexaose I from the only other known naturally occurring difucohexaose, lacto- $N$-difucohexaose II (25).

(b) Lacto- $N$-fucopentaose I gives a positive ElsonMorgan test for $N$-acetylaminosugars, whereas lacto- $N$ difucohexaose I does not. This fact was used by Kuhn and Gauhe (25) as proof that the $N$-acetyl-D-glu-

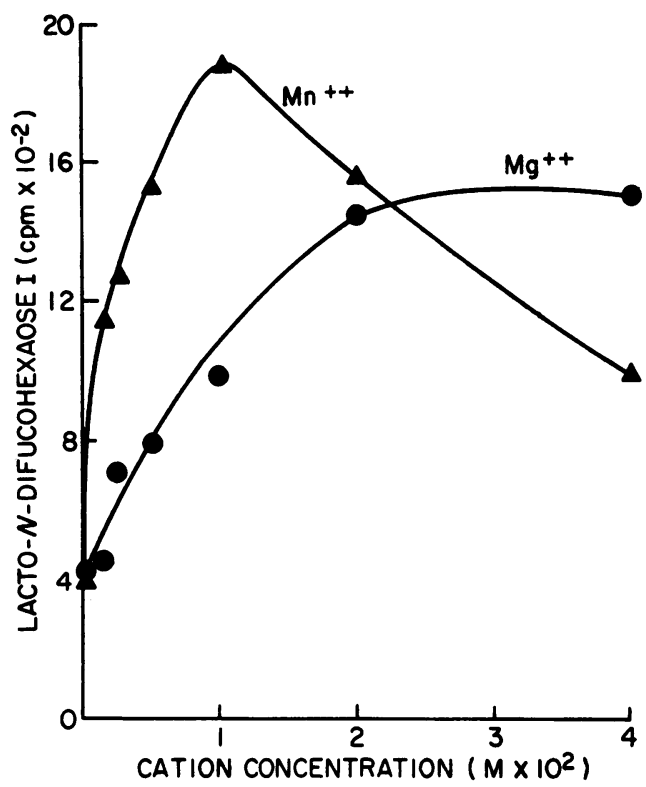

FIgURE 4 Effect of $\mathrm{MnCl}_{2}$ or $\mathrm{MgCl}_{2}$ on the formation of lacto- $N$-difucohexaose I. Reaction mixtures and conditions were the same as in Fig. 1, except that the concentration of metal ion was varied as indicated. 


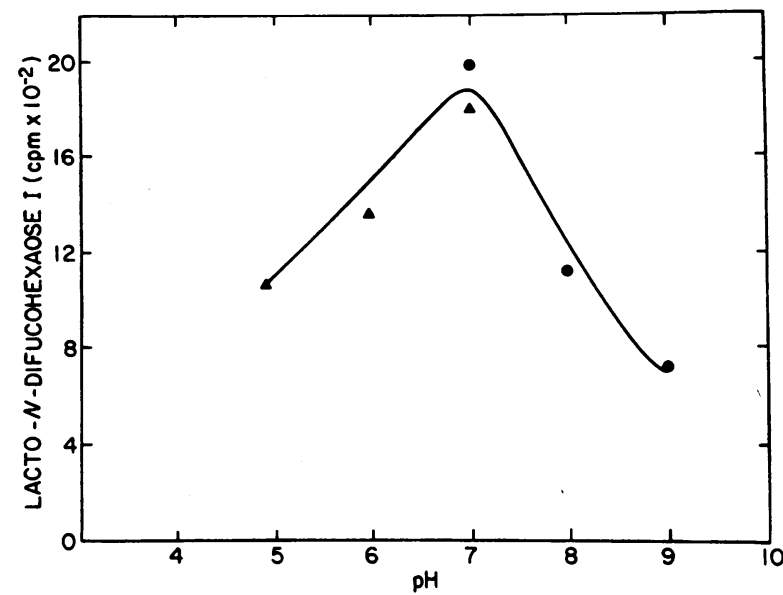

FIGURE 5 The effect of $\mathrm{pH}$ on the formation of lacto- $N$ difucohexaose $\mathrm{I}$. The reaction mixtures and conditions were the same as in Fig. 1, except that $2.5 \mu$ moles of the following buffers of varying $\mathrm{pH}$ were used: - - -1 , Tris-hydrochloride; $\mathbf{A}-\mathbf{A}-\mathbf{\Delta}$, Tris-maleate.

cosamine residue of lacto- $N$-difucohexaose I was substituted in the 4-position with L-fucose, as $N$-acetylhexosamines do not react in this test when they are substituted in the 4-position (27). The characteristic double-peaked absorption spectrum exhibited by lacto$N$-fucopentaose I in the Elson-Morgan test is shown in Fig. 2. Lacto- $N$-difucohexaose $\mathrm{I}$ as well as the product of the enzymatic reaction did not react with this reagent.

(c) Lacto- $N$-difucohexaose $I$ is a potent and highly specific haptenic inhibitor of the quantitative precipitin reaction between human $\mathrm{Le}^{\mathrm{b}}$ substance and goat $\mathrm{Le}^{\mathrm{b}}$ antiserum (28). $6 \mu \mathrm{g}$ of the reaction product inhibited the precipitin reaction to the same extent as $4 \mu \mathrm{g}$ of authentic lacto- $N$-difucohexaose I. In this test, no inhibition was observed with up to $62 \mu \mathrm{g}$ of lacto- $N$-fucopentaose I or lacto- $N$-difucohexaose II (28).

All of the above results indicate that the reaction product is lacto- $N$-difucohexaose $\mathrm{I}$, and hence the enzyme of Table II can be described as a GDP-L-fucose: $N$-acetyl-D-glucosaminylsaccharide $\alpha$-4-L-fucosyltransferase.

Properties of the enzyme. The proportionality of the reaction with time and with enzyme concentration is shown in Fig. 3. Under the conditions of assay the reaction is linear for at least $8 \mathrm{hr}$ and directly proportional to enzyme concentration. Both $\mathrm{Mg}^{++}$and $\mathrm{Mn}^{++}$stimulate the reaction as shown in Fig. 4. The $\mathrm{pH}$ optimum for the reaction is approximately 7.0 (Fig. 5) which is the same as that reported for lactose synthetase (29). The effect of increasing concentrations of lacto- $N$-fucopentaose I on the synthesis of lacto- $N$-difucohexaose I is shown in Fig. 6. From the reciprocal plot given in the

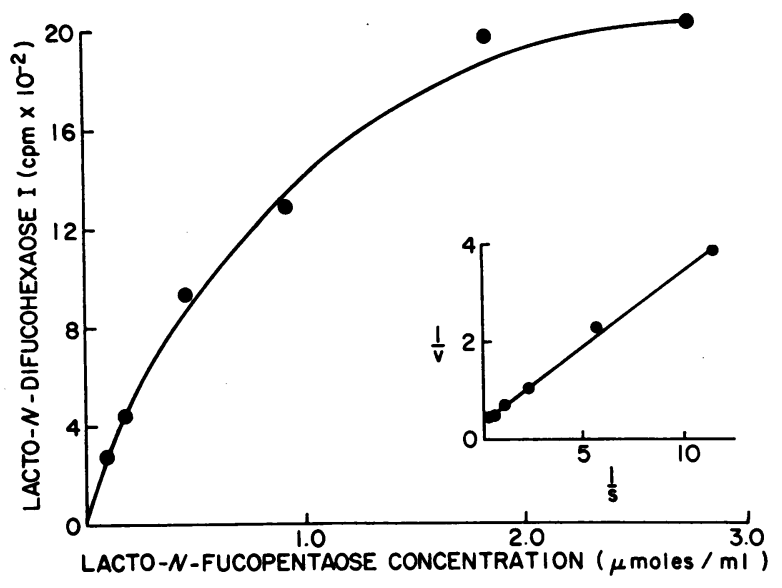

Figure 6 The effect of lacto- $N$-fucopentaose I concentration on the formation of lacto- $N$-difucohexaose $\mathrm{I}$. The reaction mixtures and conditions were the same as in Fig. 1 , except that the concentration of lacto- $N$-fucopentaose I was varied as indicated.

inset, the $K_{m}$ for lacto- $N$-fucopentaose I under the conditions of incubation is $8.3 \times 10^{-4}$.

\section{DISCUSSION}

The presence or absence of specific glycosyltransferases appears responsible for the genetic control of cellular antigens whose structural determinants are carbohydrates $(9,30-33)$. Thus, the antigens can be considered to be secondary gene products, in that the primary products are enzymes and it is these enzymes working in concert that determine which specific structures are formed. This mechanism of synthesis provides a biochemical explanation for gene interaction, i.e., for the production of antigens peculiar to the hybrid and absent in both homozygous parents. The Le $\mathrm{L}^{\mathrm{b}}$ determinant is one of these. On the basis of family studies Ceppellini proposed that the $\operatorname{Le}(\mathrm{b}+)$ character was an interaction product of two genes (10) which, in the scheme of Watkins and Morgan, would be the $H$ gene (whose expression in certain secretory organs is controlled by the independently inherited $S e$ gene) and the $L e$ gene and would produce the enzymes responsible for the incorporation into the antigenic structure of the two fucose residues that are required for $\mathrm{Le}^{\mathrm{b}}$-active structures $(11,12)$. The $H$ gene would produce the fucosyltransferase described in a previous publication (9), whereas the $L e$ gene would produce the fucosyltransferase of this publication. The product of the combined action of these two enzymes is a serologic determinant that is not formed by either alone, and if each parent provided the gene for only one of the two enzymes, then the determinant would clearly be a "gene interaction product." In addition to the Lewis $(b+)$ character there are several reports of 
interaction products of other cellular antigens including interactions within the $\mathrm{Rh}$ system and interactions of the $I$ gene with the $A, B$, or $H$ genes (cf. reference 14). The formation of unique carbohydrate structures by the concerted action of glycosyltransferases provided by both parents may be a factor in the expression of individuality and involved in various phenomena of cell recognition such as graft rejection.

\section{ACKNOWLEDGMENT}

We are indebted to Dr. Donald Marcus of The Albert Einstein College of Medicine for performing the haptemic inhibition studies.

\section{REFERENCES}

1. Grollman, E. F., A. Kobata, and V. Ginsburg. 1968. An enzymatic basis for Le(a-b-) blood type in humans. Fed. Proc. $27: 345$.

2. Mourant, A. E. 1946. A 'new' human blood group antigen of frequent occurrence. Nature (London). 158: 237.

3. Andresen, P. H. 1948. The blood group system L. A new blood group $\mathrm{L}_{2}$. A case of epistasy within the blood groups. Acta Pathol. Microbiol. Scand. 25: 728.

3a. Andersen, P. H. 1961. Relations between the ABO, secretor/non-secretor, and Lewis systems with particular reference to the Lewis system. Amer. J. Hum. Genet. 13: 396.

4. Kabat, E. A. 1956. Blood Group Substances. Academic Press Inc., New York. 15.

5. Watkins, W. M., and W. T. J. Morgan. 1962. Further observations on the inhibition of blood-group specific serological reactions by simple sugars of known structure. Vox. Sang. 7: 129.

6. Rege, V. P., T. J. Painter, W. M. Watkins, and W. T. J. Morgan. 1964. Isolation of a serologically active, fucose containing, trisaccharide from human blood-group $\mathrm{Le}^{\star}$ substance. Nature (London). 204: 740.

7. Lloyd, K. O., E. A. Kabat, and E. Licerio. 1968. Immunochemical studies on blood groups XXXVIII. Structures and activities of oligosaccharides produced by alkaline degradation of blood-group Lewis ${ }^{\mathrm{a}}$ substance. Proposed structure of the carbohydrate chains of human blood-group $\mathrm{A}, \mathrm{B}, \mathrm{H}, \mathrm{Le}^{\mathrm{a}}$ and $\mathrm{Le}^{\mathrm{b}}$ substances. Biochemistry. 7: 2976.

8. Marr, A. M. S., A. S. R. Donald, W. M. Watkins, and W. T. J. Morgan. 1967. Molecular and genetic aspects of human blood-group Le ${ }^{\mathrm{b}}$ specificity. Nature (London). 215: 1345.

9. Shen, L., E. F. Grollman, and V. Ginsburg. 1968. An enzymatic basis for secretor status and blood group substance specificity in humans. Proc. Nat. Acad. Sci. $U . S .59: 224$.

10. Ceppellini, R. 1954. On the genetics of secretor and Lewis characters: a family study. Proceedings of the 5th International Congress of Blood Transfusion, Paris. 207.

11. Watkins, W. M., and W. T. J. Morgan. 1959. Possible genetical pathways for the biosynthesis of blood group mucopolysaccharides. Vox. Sang. 4: 97.

12. Watkins, W. M. 1966. Glycoproteins. A. Gottschalk, editor. Elsevier, Amsterdam. 462.

13. Ginsburg, V. 1966. Methods in Enzymology. E. F. Neu- feld and V. Ginsburg, editors. Academic Press Inc., New York. 8: 145.

14. Race, R. R., and R. Sanger. 1962. Blood groups in man. Blackwell Scientific Publications, Ltd., Oxford.

15. Anet, E. F. L. J., and T. M. Reynolds. 1954. Isolation of mucic acid from fruits. Nature (London). 174: 930.

16. Dische, Z., and L. B. Shettles. 1948. A specific color reaction of methylpentoses and a spectrophotometric micromethod for their determination. J. Biol. Chem. 175 595.

17. Kobata, A., M. Tsuda, and V. Ginsburg. 1969. Oligosaccharides of human milk. I. Isolation and characterization. Arch. Biochem. Biophys. 130: 509.

18. Elson, L. A., and W. T. J. Morgan. 1933. A colorimetric method for the determination of glucosamine and chondrosamine. Biochem. J. 27: 1824.

19. Grollman, E. F., and V. Ginsburg. 1967. Correlation between secretor status and the occurrence of 2 -fucosyllactose in human milk. Biochem. Biophys. Res. Commun. 28: 50 .

20. Kuhn, R., H. H. Baer, and A. Gauhe. 1958. Die Konstitution der Lacto- $N$-fucopentaose II; ein Beitrag zur Spezifität der Blutgruppensubstanz Le ${ }^{\mathrm{a}}$. Chem. Ber. 91: 364.

21. Kuhn, R., H. H. Baer, and A. Gauhe. 1956. Kristallisation und Konstitutionsermittlung der Lacto- $N$-fucopentaose I. Chem. Ber. 89: 2515.

22. Kuhn, R. 1958. Les oligosaccharides du lait. Bull. Soc. Chim. Biol. 40: 297.

23. Kuhn, R., H. H. Baer, and A. Gauhe. 1958. 2- $\alpha-\mathrm{L}-$ Fucopyranosyl-D-galaktose und 2- $\alpha$-L-Fucopyranosyl-Dtalose. Zur Einwirkung von Alkali auf Oligosaccharide. Justus Liebigs. Anal. Chem. 611: 242.

24. Grubb, R., and W. T. J. Morgan. 1949. The "Lewis" blood group characters of erythrocytes and body-fluids. Brit. J. Exp. Pathol. 30: 198.

25. Kuhn, R., and A. Gauhe. 1960. Über ein Kristallisiertes, Le $^{a}$-aktives Hexasaccharid aus Frauenmilch. Chem. Ber. 93: 647 .

26. Montreuil, J. 1960. Glucides du lait. Bull. Soc. Chim. Biol. 42: 1399

27. Kuhn, R., A. Gauhe, and H. H. Baer. 1954. Einfluss von Substituenten auf die Farbreaktion von $N$-Acetylglucosamin mit $p$-Dimethylaminobenzaldehyd. Chem. Ber. 87: 1138

28. Marcus, D. M., and A. P. Grollman. 1966. Studies on blood group substances. I. Caprine precipitating antisera to human $\mathrm{Le}^{\mathrm{a}}$ and $\mathrm{Le}^{\mathrm{b}}$ blood group substances. J. Immunol. $97: 867$.

29. Babad, H., and W. Z. Hassid. 1966. Soluble uridine diphosphate D-galactose: D-glucose $\beta$-4-D-galactosyltransferase from bovine milk. J. Biol. Chem. 241: 2672.

30. Kobata, A., E. F. Grollman, and V. Ginsburg. 1968. An enzymic basis for blood type $\mathrm{A}$ in humans. Arch. Biochem. Biophys. 124: 609 .

31. Kobata, A., E. F. Grollman, and V. Ginsburg. 1968. An enzymatic basis for blood type $\mathrm{B}$ in humans. Biochem. Biophys. Res. Commun. 32: 272.

32. Race, C., D. Ziderman, and W. M. Watkins. 1968. An $\alpha$-D-galactosyltransferase associated with the blood-group B character. Biochem. J. $107: 733$.

33. Carlson, D. M. 1968. II. Studies on pig submaxillary mucin. In Biochemistry of Glycoproteins and Related Substances. E. Rossi and E. Stoll, editors. S. Karger, AG, New York. 